\title{
Alfabetización informacional en la modalidad blended learning en educación superior
}

\author{
André Armel Maguiña Ballón \\ Universidad Peruana de Ciencias Aplicadas - UPC, Perú
}

\section{REVIEW}

\begin{abstract}
Resumen
Objetivo. El objetivo general es analizar la aplicación de la alfabetización informacional en la enseñanza y el aprendizaje de la educación superior según la modalidad blended, a partir de la revisión de la literatura especializada en las bases de datos bibliométricas Web of Science y Scopus.

Método. Se revisaron 18 documentos como resultado de la aplicación de una metodología que incluyó la definición de términos de búsqueda y de variables, criterios de exclusión, estandarización y elaboración de las preguntas de investigación. Las categorías a analizar se organizaron según los objetivos específicos y son las siguientes: modelos y estándares, modalidades de enseñanza y aprendizaje, entornos tecnológicos y disciplinas, sin ninguna restricción geográfica o temporal.

Resultados. Los resultados muestran que la mayoría de los modelos y estándares utilizados son propios de la alfabetización informacional, en especial los establecidos por la Association of College and Research Libraries (2000 y 2015); en tanto que solo tres son generales e incluyen algún aspecto de la alfabetización informacional. Asimismo, la mayoría de los documentos incluyen investigaciones en cursos o programas académicos, seguidos de estudios sobre sistemas de gestión de aprendizaje. Los entornos tecnológicos predominantes son los sistemas de gestión del aprendizaje y los entornos virtuales de aprendizaje en línea. Finalmente, las disciplinas son diversas, entre las cuales figuran con más investigaciones las ciencias de la información, inglés, educación y tecnología de la información.

Conclusiones. El aprendizaje blended implica un desafío para la aplicación de la alfabetización informacional en la educación superior, en especial, en cuanto a los diseños instruccionales de los cursos o de los programas académicos, los modelos pedagógicos que los respaldan y los entornos tecnológicos adecuados.
\end{abstract}

Palabras clave:

Alfabetización informacional; Aprendizaje mixto; Blended learning; Desarrollo de habilidades informativas; Educación superior

\section{Literature review: information literacy in blended learning in higher education}

\section{Abstract}

Objetive. The main objective is to analyze the application of information literacy in the teaching and learning of higher education according to the blended modality, based on the review of specialized literature in the Web of Science and Scopus bibliometric databases.

Method. 18 documents were reviewed as a result of the application of a methodology that included the definition of search terms and variables, exclusion criteria, standardization and elaboration of research questions. The categories to be analyzed were organized according to the specific objectives and are the following: models and standards, teaching and learning modalities, technological environments and disciplines, without any geographical or temporal restrictions.

Results. The results show that most of the models and standards used are typical of information literacy, especially those established by the Association of College and Research Libraries (2000 and 2015); while only three are general and include some aspect of information literacy. Also, most documents include research in courses or academic programs, followed by studies on learning management systems. The predominant technological environments are learning management systems and virtual online learning environments. Finally, the disciplines are diverse, among which the information sciences, English, education, and information technology stand out with more research.

Conclusions. Blended learning implies a challenge for the application of information literacy in higher education, especially in terms of the instructional designs of courses or academic programs, the pedagogical models that support them and the appropriate technological environments.

Keywords:

Blended learning; Higher education; Information literacy; Information skills; Information problem solving. 


\section{Introducción}

Una de las características de la cultura digital es la utilización de artefactos denominados tecnologías de la información y de la comunicación (TIC), tecnologías digitales e infraestructura de internet. La integración de estas tecnologías a la cultura de una sociedad produce impactos de todo tipo. Entre ellos se encuentran los procesos cognitivos, que implican aprender y asimilar las prácticas y los usos de esas tecnologías, aprender nuevas competencias «informacionales» y estar alfabetizado digitalmente para poder afrontar la sobreabundancia de datos e información. Además, esta cultura se caracteriza por ser interactiva y horizontal, en la cual las personas no solo consumen sino que también producen información en una sociedad red, tal como es definida por Castells (2014).

Para abordar y entender las necesidades y el uso de la información académica, surgió el concepto de alfabetización informacional, término utilizado por primera vez en 1974 por Paul Zurkowski (Lau y Cortés, 2009; Uribe, 2013) y que proviene de la traducción literal del término inglés information literacy (Calderón, 2010; Uribe, 2013). El uso de este término en la literatura -alfabetización informacional- ha ido prevaleciendo frente a otros como alfabetización informativa o alfabetización en información. Este concepto presenta numerosas definiciones y enfoques que se han ido elaborando según su evolución. Una de estas definiciones, que ha servido de referencia para otros modelos, es la de la Association of College and Research Libraries (ACRL, 2000, pp. 2) que la define como «un conjunto de habilidades que requieren los individuos para reconocer cuándo necesitan información y tienen la habilidad de localizar, evaluar y usar efectivamente la información necesitada», por lo cual, un individuo alfabetizado en información debe ser capaz de determinar el alcance de la información necesitada; acceder a dicha información en forma efectiva y eficiente; evaluar esa información en forma crítica, incorporarla en su propia base de conocimiento; usarla para un propósito específico; y entender las consideraciones éticas y legales de dicho uso (Association of College and Research Libraries (ACRL, 2000).

Este proceso de dónde y cómo evaluar la información implica la capacidad de seleccionar fuentes adecuadas y saber buscar, seleccionar y evaluar la información recuperada (Australian and New Zealand Institute for Information Literacy, 2004; Jaramillo, Henning y Rincón, 2011; Lau, 2004; REBIUN. Comisión mixta CRUE-TIC y REBIUN, 2012; SCONUL Working Group on Information Literacy, 2011).

Por otro lado, en el ámbito universitario han surgido nuevas modalidades de enseñanza y aprendizaje, así como nuevas herramientas tecnológicas que favorecen el proceso educativo. Este nuevo escenario está exigiendo que las universidades replanteen sus objetivos de enseñanza y de aprendizaje para responder a demandas de formación más flexibles, así como a contextos sociales y culturales más complejos (De Pablos, 2010). La irrupción de estas tecnologías, en especial desde el siglo XXI, ha transformado los modelos de enseñanza y de aprendizaje y ha posibilitado el surgimiento de nuevas modalidades, tales como el e-learning, el mobile leaning y el blended learning (Bartolomé, García-Ruiz, y Aguaded, 2018).

Frente al modelo tradicional de enseñanza, ha surgido el modelo mixto, híbrido, semipresencial, mezclado o blended, (Salinas, De Benito, Pérez, y Gisbert, 2018) que se desarrolla tanto en línea como en forma presencial. Sin embargo, para autores como García Aretio (2018), el origen de este concepto se remonta al inicio de la educación a distancia, especialmente en las décadas de 1960 y 1970 del siglo XX. En este sentido, según el Informe Horizon (Adams et al., 2017) el aprendizaje blended va en aumento en las universidades.

Por estas consideraciones, surge la inquietud de descubrir cómo se ha ido aplicando la alfabetización informacional en la enseñanza y el aprendizaje de la educación superior, teniendo en cuenta este nuevo contexto, cuyos «abordajes pedagógicos tradicionales pasan a no ser compatibles con los nuevos modos de pensar la educación, que se revela dirigida hacia la interactividad, la conectividad y otras dinámicas comunicacionales» (Giannasi-Kaimen, Carelli, y Gimenes da Cruz, 2010, pp. 176).

\subsection{Alfabetización informacional}

No existe consenso entre los autores acerca la terminología empleada. Algunos términos utilizados para definir este concepto son: desarrollo de habilidades informativas (DHI), usado especialmente en América Latina, habilidades informacionales (information skills), fluidez o dominio en información (information fluency), alfabetización mediática e informacional (media and information literacy) (Calderón, 2010; Pinto, 2019), alfabetización informacionalcompetencias informacionales ALFIN-COMPINFO (Uribe, 2013), competencias de manejo de la información (Jaramillo et al. 2011) y resolución de problemas de información —information problem solving - (Brand-Gruwel, Wopereis y Walraven, 2009; Eisenberg y Berkowitz, 1992; Wopereis, Frerejean y Brand-Gruwel, 2015), concepto definido como el proceso de identificar una necesidad de información, localizar, seleccionar e integrar información de diversas fuentes para resolver esa necesidad de información. Según Wopereis, Frerejean y Brand-Gruwel (2015) es un término similar al de alfetización informacional. 
Sin embargo, la mayor falta de precisión se da entre los conceptos de alfabetización informacional y otros similares. Por un lado, para algunos autores la alfabetización informacional y otros conceptos, tales como competencias informacionales o de manejo de información, son considerados como iguales o similares (González, 2012; Uribe, 2013; Wopereis et al., 2015). Por otro lado, para algunos autores y organizaciones, la alfabetización informacional es el proceso y la competencia de manejo de información - o competencia informacional— es la consecuencia o resultado de aquella (Área y Guarro, 2012; ACRL, 2000; Coonan et al., 2018; Jaramillo et al., 2011; Lau, 2004). Para la presente investigación se considera la distinción entre ambos conceptos. La alfabetización informacional alude al proceso sociocognitivo de desarrollo de habilidades informacionales para el uso de la información con el fin de resolver una necesidad de información, mientras que las competencias informacionales o de manejo de la información son el resultado de ese proceso y comprenden aquellas relacionadas con la alfabetización informacional.

Asimismo, diversas organizaciones han creado modelos, estándares, normas y declaraciones (Big6, 2017; Calderón, 2010; Crawford y Irving, 2007; Cuevas, 2007; González, 2012; Lanning, 2017; Pinto, 2019; Uribe, 2013). Para evitar la confusión terminológica acerca de los conceptos de modelos, normas y estándares, es necesario partir de las propuestas del concepto de modelo según Cuevas (2007) y Pinto (2019). Estas autoras consideran los modelos desde el punto de vista teórico. En el caso de Pinto (2019) «suponen el primer eslabón en el desarrollo de ALFIN como paradigma y disciplina académica» (p. 91), mientras que para Cueva (2007) un modelo presenta cuál es el nivel de competencias informacionales que necesita una persona en un estadio evolutivo determinado para que pueda adquirir las habilidades que lo hagan ser considerado alfabetizado en información.

Desde el punto de vista pragmático y operativo, las normas permiten el desarrollo e implementación de los modelos (Cuevas, 2007; Pinto, 2019). En cuanto a los estándares, estos remiten a las normas (Pinto, 2019), mientras que para Uribe (2013) constituyen el mismo concepto y los denomina 'normas-estándares'.

Existen algunos enfoques que se han aplicado a la alfabetización informacional con relación a contextos no solamente presenciales. Anderson y May (2010) examinan cómo la efectividad de los métodos de instrucción -en línea, blended y presencial- influyen en la capacidad de retención y uso de información en cursos básicos de pregrado. Otro modelo es el descrito por Brand-Gruwel, Wopereis y Walraven (2009), desarrollado para la resolución de problemas de información (information problem solving) aplicado a internet, al cual denominan IPS-I-model y que consta de cinco habilidades: definición del problema de información; búsqueda de información; información de escaneo (scanning information); información de procesamiento: y organización y presentación de la información.

\subsection{La modalidad de enseñanza y aprendizaje blended en educación superior}

Como ya se mencionó anteriormente, esta modalidad de aprendizaje tiene varias denominaciones. Sin embargo, el término blended learning es el más utilizado en la literatura en español según los resultados de Google Scholar, muy por encima de 'aprendizaje mixto' (Bartolomé et al., 2018). Por esta razón, este será el término que se utilizará en la presente investigación.

Los alcances del aprendizaje blended son considerables y su flexibilidad, su facilidad de acceso y la integración de los recursos digitales con las tecnologías de la información son cada vez más apreciados. Gros, García y Escofet (2012) destacan el valor de las TIC en relación con el proceso de aprendizaje, más que con el contenido. Nortvig, Petersen y Balle (2018) analizan algunos factores que influyen en el aprendizaje electrónico (e-learning) y el blended, tales como la presencia del docente en la configuración del entorno en línea, las interacciones entre los estudiantes, los docentes y el contenido, y las conexiones diseñadas entre las actividades en línea y fuera de línea (offline). En esta línea, Singer y Stoicescu (2011) resaltan el estímulo del pensamiento crítico y la promoción de la participación de los estudiantes.

En cuanto a la modalidad flipped learning o aprendizaje invertido se trata de una aproximación educativa en la cual se invierte el método de enseñanza tradicional, otorgando a los estudiantes los contenidos en forma electrónica para que los revisen antes de las clases presenciales, en las cuales se utiliza el tiempo en forma práctica, ya sea resolviendo ejercicios o problemas, discutiendo ejemplos, realzando actividades colaborativas o de interacción (Chen, Ritzhaupt y Antonenko, 2018; Kurbanoĝlu y Akkoyunlu, 2016).) Existen diversas investigaciones sobre esta modalidad. Arnold-Garza (2014) describe algunas características de este modelo que pueden ser utilizadas para la aplicación pedagógica de la alfabetización informacional en educación superior, en tanto que Rodríguez (2016) explica cómo las sesiones de alfabetización informacional para clases con gran cantidad de estudiantes matriculados pueden ser adaptadas al modelo flipped learning e incorporar actividades de aprendizaje activo. 


\subsection{Justificación y objetivos}

Una aproximación a la literatura parece sugerir que no existe suficiente investigación sobre ambos conceptos en educación superior. Si bien una búsqueda precisa en Google Académico recupera una gran cantidad de resultados, estos no necesariamente se refieren a investigaciones sobre la alfabetización informacional únicamente en blended learning, sino en diferentes modalidades -e-learning, presencial o ambas-, así como otros temas similares. Situación diferente se da en cuanto a las investigaciones que registran las dos principales bases de datos que miden la calidad de las publicaciones científicas según diferentes criterios y métricas, es decir, Web of Science y Scopus. En este sentido, teniendo en cuenta el vacío que existe en la literatura indizada en Scopus y Web of Science, se tiene la siguiente pregunta de investigación: ¿cómo se ha aplicado la alfabetización informacional en educación superior en la modalidad blended learning a partir de la literatura especializada? Las categorías para analizar son las siguientes: modelos, modalidades de enseñanza y aprendizaje, entornos tecnológicos y disciplinas.

El objetivo general es el siguiente: analizar la aplicación de la alfabetización informacional en educación superior en la modalidad blended learning, a partir de la literatura especializada en dos bases de datos.

Los objetivos específicos son los siguientes:

- Identificar los principales modelos de alfabetización informacional utilizados en la modalidad blended en educación superior.

- Determinar a través de qué modalidades de enseñanza y aprendizaje se ha aplicado la alfabetización informacional en la educación superior de tipo blended.

- Identificar qué entornos tecnológicos se han utilizado en la alfabetización informacional en la modalidad blended en educación superior.

- Reconocer en qué disciplinas se ha aplicado la alfabetización informacional en la modalidad blended en educación superior.

\section{Metodología}

El presente estudio constituye un artículo de revisión, cuya finalidad es «examinar la bibliografía publicada anteriormente y situarla en cierta perspectiva» (Day, 2005, pp. 158). Se trata de una revisión descriptiva, debido a que constituye «una puesta al día sobre conceptos útiles en áreas en constante evolución» (Merino-Trujillo, 2011, pp. 36).

La justificación para la elección de Scopus y Web of Science está en concordancia con López-Meneses, VázquezCano y Román (2015). Estos autores afirman que el uso comparado de bases de datos, como Web of Science, Scopus y Google Scholar, es un método usado para medir cuál es el impacto de un término o de una tendencia. Varios autores coinciden al respecto (Doğan, Şencan y Tonta, 2016; Jacso, 2005; Martín-Martín, Orduna-Malea y Delgado López-Cózar, 2018; Martín-Martín, Orduna-Malea, Thelwall y Delgado López-Cózar, 2018; Shah, Mahmood y Hameed, 2017). Y, más recientemente, son incoporadas métricas de rankings por relavancia, incluidas en el concepto de optimización de motores de búsqueda académicos (academic search engine optimization, ASEO, por sus siglas en inglés) (Rovira, Codina, Guerrero-Solé y Lopezosa, 2019).

Sin embargo, algunos autores desconfían de los resultados presentados en Google Scholar. La manipulación de datos de citas es examinada por Delgado López-Cózar, Robinson-García y Torres-Salinas (2014), en tanto que la existencia de errores es identificada en la revisión de Orduna-Malea, Martín-Martín y Delgado López-Cózar (2017). Al respecto, Doğan, Şencan y Tonta (2016) se refieren a los "datos sucios" como las publicaciones y citaciones duplicadas que son indexadas por Google Scholar. Asimismo, y al igual que Martín-Martín, Orduna-Malea, Thelwall y Delgado López-Cózar (2018), se alerta sobre las fuentes que son citadas y no son académicas -tesis, libros y otros tipos de documentos.

Por esta razón, se justifica el uso de las dos bases bibliométricas -Web of Science y Scopus-, teniendo en cuenta que la presente revisión constituye un primer acercamiento al estudio de la alfabetización informacional en educación superior de tipo blended; y que es necesario asegurar la fiabilidad de las fuentes recuperadas. Sin embargo, queda abierta la posibilidad de un estudio futuro más integrador con Google Scholar.

Se realizó una revisión de la literatura en las bases de datos Web of Science y Scopus, con el objetivo de analizar cómo se ha aplicado la alfabetización informacional en la educación superior de pregrado según la modalidad blended. 


\subsection{Definición de términos de búsqueda}

Se realizó una búsqueda de los principales términos o palabras clave que comprendían de la mejor manera los principales conceptos de la investigación y que fueron validados por especialistas. La estrategia de búsqueda en español fue la siguiente: ("blended learning" OR "aprendizaje mixto" OR "aprendizaje invertido" OR "aprendizaje semipresencial" OR "aprendizaje híbrido") AND ("alfabetización informacional” OR "alfabetizacion informativa” OR "habilidades informacionales" OR "habilidades informativas" OR "resolución de problemas de información") AND ("educación superior" OR "universidades").

La estrategia de búsqueda en inglés fue la siguiente: "blended learning" AND ("information literacy" OR "information problem-solving" OR "information skills" OR "information fluency") AND "higher education". Los campos de búsqueda fueron los siguientes: Article title, Abstract, Keywords (TITLE-ABS-KEY) -para Scopus- y Tema (TS) -para Web of Science.

\subsection{Búsqueda y definición de la muestra}

Se realizó una búsqueda final en ambas bases de datos durante la primera semana de octubre de 2019 y se recuperaron 21 resultados en Scopus y 5 en Web of Science, todos ellos en inglés. De los 26 resultados, cuatro fueron duplicados, tres eran revisiones de conferencias y uno no trataba el análisis de la alfabetización informacional, por lo que fueron excluidos, quedando finalmente 18 resultados. Debido a la poca cantidad de resultados recuperados, se decidió no incluir criterios de exclusión ni limitar la búsqueda por período o alcance geográfico. El rango de años de publicación va desde 2006 hasta 2019.

Los países sobre los que realizaron las investigaciones pertenecen son los siguientes: Estados Unidos (3), China (2), Alemania (2), Tailandia (1), Taiwán (1), Chile (1), Sudáfrica (1), Polonia (1), Japón (1) y Reino Unido (1), en tanto que en tres estudios no se pudo identificar el país.

Los tipos de documentos son los siguientes: artículos de revistas (12), papers de conferencias (3) y capítulos de libros (3). La relación de los estos documentos de investigación se encuentra en el Anexo 1.

\subsection{Definición de variables y estandarización}

A continuación, se explica el procedimiento de estandarización de las variables a partir de los objetivos específicos.

Objetivo específico 1: identificar los principales modelos y estándares de alfabetización informacional utilizados en la modalidad blended en educación superior.

- Variable: modelos y estándares de alfabetización informacional. Para la definición de estas variables se consideran los conceptos de Cuevas (2007) y Pinto (2019), referidos en el punto 1.1: un modelo muestra, desde un punto de vista más teórico, el nivel de competencias informacionales en un estadio determinado para la adquisición de las habilidades que hacen a una persona ser considerada alfabetizada en información. En este sentido, se puede considerar a un modelo como un esquema teórico de un sistema o de una realidad compleja (Real Academia Española, 2020). Los estándares constituyen reglas, principios o criterios a través de los cuales se miden o juzgan los niveles o grados de adecuación, aceptabilidad, cantidad, calidad o valor (Institute of Education Sciences, 2019). Mientras que los estándares remiten a las normas, estas permiten el desarrollo e implementación de la alfabetización informacional (Pinto, 2019).

- Estandarización: determinar si se trata de modelos o estándares.

Objetivo específico 2: determinar a través de qué modalidades de enseñanza y aprendizaje se ha aplicado la alfabetización informacional en la educación superior de tipo blended.

- Variable: modalidades de enseñanza y aprendizaje. Comprenden el objeto de estudio sobre el cual se ha aplicado o abordado de alguna forma la alfabetización informacional en el entorno blended.

- Estandarización: precisar si se trata de un curso, un programa académico, un proyecto piloto educativo, una propuesta de modelo de alfabetización informacional, una propuesta de diseño curricular, sistemas de gestión de aprendizaje, herramientas tecnológicas en línea, entre otras modalidades.

Objetivo específico 3: identificar qué entornos tecnológicos se han utilizado en la alfabetización informacional en la modalidad blended en educación superior. 
- Variable: entornos tecnológicos. Se refiere a aquellos ambientes virtuales para el aprendizaje, sistemas de información en línea, recursos digitales en línea, aplicaciones web y otras herramientas tecnológicas.

- Estandarización: determinar qué entornos tecnológicos se han utilizado, tales como sistemas de gestión del aprendizaje (LMS, por sus siglas en inglés), aplicaciones web específicas, recursos educativos en línea, redes sociales, dispositivos utilizados, mundos virtuales, entre otros.

Objetivo específico 4: reconocer en qué disciplinas se ha aplicado la alfabetización informacional en la modalidad blended en educación superior.

- Variable: Disciplinas. También definidas como áreas del conocimiento.

- Estandarización: determinar las disciplinas en las que se desarrollan las investigaciones.

\section{Resultados}

Se presentan los resultados obtenidos como consecuencia del análisis de los 18 documentos que constituyen la población del estudio, organizados según los cuatro objetivos específicos.

3.1. Objetivo específico 1: identificar los principales modelos de alfabetización informacional utilizados en la modalidad blended en educación superior

Los resultados muestran que la gran mayoría corresponden a modelos (13) y solo dos a estándares: Psychology Information Literacy Standards, (ACRL, 2010) e Information Literacy Competency Standards for Higher Education (ACRL, 2000), este último también considerado un modelo por Pinto (2019).

A continuación, se muestran los resultados de los modelos y estándares según cuatro categorías: estándares propios de la alfabetización informacional, modelos propios de la alfabetización informacional, modelos generales que incluyen algún aspecto de alfabetización informacional y modelos que no son propios de la alfabetización informacional.

La tabla 1 muestra los dos estándares de alfabetización informacional y los documentos de investigación asociados.

Tabla 1. Estándares de alfabetización informacional.

\begin{tabular}{|l|c|}
\hline \multicolumn{1}{|c|}{ Estándares de alfabetización informacional } & Documentos \\
\hline $\begin{array}{l}\text { Information Literacy Competency Standards for Higher } \\
\text { Education (ACRL 2000) }\end{array}$ & $\begin{array}{c}\text { Chaiyama (2013) } \\
\text { Hess (2017) }\end{array}$ \\
\hline $\begin{array}{l}\text { Estándares de Alfabetización Informacional Especí- } \\
\text { ficos de Psicología (Psychologij (2019) } \\
\text { Standards, ACRL 2010) }\end{array}$ & $\begin{array}{c}\text { Kleineldt y Zulu (2016) } \\
\text { Sult y Mills (2006) }\end{array}$ \\
\hline
\end{tabular}

En la tabla 2 se presentan los modelos de alfabetización informacional y los documentos de investigación asociados.

Tabla 2. Modelos de alfabetización informacional

\begin{tabular}{|l|c|}
\hline \multicolumn{1}{|c|}{ Modelos de alfabetización informacional } & Documentos \\
\hline $\begin{array}{l}\text { ACRL Framework for Information Literacy for Higher Edu- } \\
\text { cation (2015) }\end{array}$ & $\begin{array}{c}\text { Hess (2017) } \\
\text { Mortimore y Baker (2019) }\end{array}$ \\
\hline $\begin{array}{l}\text { Modelo de gestión de aprendizaje blended para desarro- } \\
\text { llar habilidades de alfabetización informacional (Blen- } \\
\text { ded-learning management model in developing informa- } \\
\text { tion literacy skills for student) }\end{array}$ & Chaiyama (2013) \\
\hline Modelo Big 6 Skills & Chaiyama (2013) \\
\hline $\begin{array}{l}\text { Modelo de mejora de la alfabetización informacional para } \\
\text { la sociedad tailandesa (Information Literacy Enhance- } \\
\text { ment model for Thai Society) }\end{array}$ & Chaiyama (2013) \\
\hline
\end{tabular}




\begin{tabular}{|l|c|}
\hline $\begin{array}{l}\text { Modelo: Blended Learning Model of Information Techno- } \\
\text { logy Education }\end{array}$ & Mei, Qi y Zhang (2016) \\
\hline $\begin{array}{l}\text { Método: Information Technology Education Blended } \\
\text { Learning Mode Based on the Flipped Classroom }\end{array}$ & Mei et al. (2016) \\
\hline $\begin{array}{l}\text { Método: Information Technology Education Blended al. (2016) } \\
\text { Learning Mode Based on the Resource Utilization }\end{array}$ & Mei et al. (2016) \\
\hline $\begin{array}{l}\text { Método: Information Technology Education Blended } \\
\text { Learning Mode Based on Project Learning }\end{array}$ & Heinze y Schnurr (2008) \\
\hline $\begin{array}{l}\text { Modelo de andamiaje heurístico dentro del ciclo de vida } \\
\text { del estudiante }\end{array}$ & Tonakiewicz-Kołosowska, Socik y Gajewska (2016) \\
\hline National Qualifications Framework &
\end{tabular}

Fuente: elaboración propia

Destacan en primer lugar con seis investigaciones los estándares de la ACRL (2000), Information Literacy Competency Standards for Higher Education, que constituyen cinco estándares de competencia informacional y 22 indicadores de desempeño para estudiantes de educación superior, con el objetivo de evaluar el progreso del estudiante durante el desarrollo de la alfabetización informacional. Basados en los estándares de la ACRL para la educación superior (2000), los Estándares de Alfabetización Informacional Específicos de Psicología (Association of College and Research Libraries [ACRL], 2010) fueron utilizados por Peter et al. (2017) para ajustar los contenidos en línea en el diseño instruccional de un curso blended, en especial en los procesos de búsqueda, acceso y evaluación de la información en un contexto académico —estándares 2 y 3.

Esta misma institución actualizó los estándares del año 2000 hacia un marco de referencia de tipo más integrador u holístico, denominado Framework for Information Literacy for Higher Education, que consiste en una serie de conceptos centrales interconectados que funciona como vías para la comprensión del pensamiento y la práctica dentro de cualquier disciplina. Estos conceptos están organizados en seis marcos e incorporan prácticas de conocimiento y disposiciones, es decir, cómo abordar las dimensiones afectivas, actitudinales o de valor del aprendizaje. Estos seis marcos incorporan los siguientes conceptos: la autoridad es construida y es contextual; la creación de información es un proceso; la información tiene valor; la investigación es una indagación: el aprendizaje es un diálogo; y la búsqueda de información es una exploración estratégica (Association of College and Research Libraries [ACRL], 2015). Aunque el documento se titula 'marco de referencia' y no 'modelo', algunos autores como Pinto (2019) lo consideran como un nuevo modelo de alfabetización informacional. Si bien incorpora conceptos centrales flexibles organizados en seis marcos relacionados con sus respectivas disposiciones y prácticas de conocimiento, se puede considerar que esa estructura representa el esquema teórico de un sistema, (aunque no de una realidad), lo cual se ajustaría al concepto de modelo, tal como se explicó en el punto 2.3.

Otro modelo de carácter general y comúnmente aceptado es el denominado Big6 (2017), creado originalmente por Mike Eisenberg y Bob Berkowitz en 1990. Este modelo es considerado por Chaiyama (2013) para el desarrollo de su modelo de gestión de habilidades de alfabetización informacional para el aprendizaje blended. Big6 se define como un modelo de seis etapas basado en la solución de problemas de información —information problemsolving-y en las habilidades requeridas para resolverlos.

Por otro lado, la tabla 2 muestra investigaciones sobre propuestas de modelos o sobre modelos de aplicación más específica.

En el primer caso, el ya mencionado estudio de Chaiyama (2013) propone un modelo de gestión de habilidades de alfabetización informacional para el aprendizaje blended. Este modelo implica un proceso continuo de gestión del aprendizaje en dos fases: la preparación de la actividad presencial y la realización de las estrategias de gestión del aprendizaje para desarrollar habilidades de alfabetización informacional, con actividades tanto presenciales como en línea. En dicho modelo se enfatiza el aprendizaje de los estudiantes en forma grupal y el rol de instructores como facilitadores para guiar el aprendizaje. Finalmente, el modelo incorpora secuencialmente las siguientes etapas basadas en el modelo Big6: determinación de la tarea para el estudio; determinación de estrategias para buscar información; búsqueda y recopilación de información; análisis, síntesis y organización de la información; y evaluación y presentación de la información.

Mei et al. (2016), al igual que Chaiyama, proponen un modelo general para el aprendizaje blended aplicado a cursos de tecnología de la información, pensado como un proceso continuo, según los diferentes niveles de los estudiantes. El modelo se compone de tres capacidades a lograr: conocimiento y cultura de la tecnología multimedia; habilidades y aplicación de la tecnología de la información; y método e innovación de la tecnología de 
la información. Cada una de estas capacidades comprende una estructura del conocimiento que precisa los temas centrales que les corresponde, así como su relación con las asignaturas del sistema curricular. Basados en este modelo general, estos autores establecen tres modos de aprendizaje blended, según la modalidad definida. El primero está orientado al aprendizaje invertido o flipped learning, el segundo está basado en el uso de los recursos pedagógicos y el diseño instruccional que lo respalda, y el tercero se refiere al aprendizaje basado en proyectos.

Otro modelo propuesto es el de andamiaje heurístico dentro del ciclo de vida del estudiante (Heinze y Schnurr, 2008), el cual integra diferentes niveles de competencias con diversos elementos didácticos a lo largo del ciclo de vida del estudiantes, de acuerdo con cinco fases: en la primera se ofrece información básica sobre estrategias del trabajo científico; en la segunda se aprende métodos de investigación y escritura académica; en la tercera se aborda la planificación y redacción de la tesis de bachillerato; en la cuarta, dirigida a estudiantes de maestría, se profundizan algunos contenidos teoréticos; y en la quinta se apoya la elaboración de la tesis de maestría.

En cuanto a modelos de aplicación más específica, Chaiyama (2013) incorpora un modelo que aspira a ser implementado a nivel de políticas de Estado. A pesar de ser una propuesta, pretende mejorar la alfabetización informacional para la sociedad tailandesa. No obstante, no se ha podido acceder al contenido del estudio ni se ha encontrado documentación que respalde su adopción oficial, lo cual parece confirmarse con los hallazgos de Tuamsuk (2013) que mencionan solamente los estándares de la ACRL (2000) y el modelo de SCONUL (2011) en la educación superior tailandesa.

Otra categoría de modelos son aquellos de carácter general y que incorporan algún tipo de proceso relacionado con la alfabetización informacional. Estos modelos se muestran en la tabla 3.

Tabla 3. Modelos generales que incluyen alfabetización informacional

\begin{tabular}{|c|c|}
\hline $\begin{array}{c}\text { Modelos generales que incluyen alfabetización } \\
\text { informacional }\end{array}$ & Documentos \\
\hline Metaalfabetización (Metaliteracy) & Ma, Li y Liang (2019) \\
\hline Modelo KSAVE & Zurita, Hasbun, Baloian y Jerez (2015) \\
\hline National Qualifications Framework & Tonakiewicz-Kołosowska et al. (2016) \\
\hline
\end{tabular}

Fuente: elaboración propia

A nivel de modelos de aplicación a nivel de Estado y, a diferencia de Chaiyama (2013), Tonakiewicz-Kołosowska et al. (2016) mencionan el Marco Polaco de Cualificaciones (National Qualifications Framework) como el contexto dentro del cual realizan su investigación. Este modelo, también denominado marco, fue establecido oficialmente en el sistema educativo polaco de educación superior en marzo de 2011 y está alineado con el Marco Europeo de Cualificaciones (MEC). Comprende ocho niveles que describen, mediante descriptores (Cedefop, 2017), los resultados de aprendizaje requeridos para cada uno de ellos. Las competencias de información son definidas como habilidades generales y divididas en competencias para estudiantes que recién empiezan —que incluyen la capacidad de buscar información en diferentes formatos-y las competencias avanzadas — que incorporan, además, evaluación e interpretación de la información.

El modelo KSAVE es un proyecto a nivel mundial (Griffin y Care, 2015; Zurita et al. 2015) cuyo objetivo es definir las habilidades del siglo XXI mediante un conjunto de conocimientos, habilidades, actitudes, y valores éticos. Tiene diez habilidades clasificadas en cuatro categorías: formas de pensar, formas de trabajo, vivir en el mundo, y herramientas para trabajar y comunicarse. Dentro de esta última se encuentra la alfabetización informacional y en TIC.

También de carácter general y de uso más reciente, el modelo de metaalfabetización o metaliteracy, aplicado por Ma et al. (2019) e incorporado por la ACRL (2015) en su Marco para la Alfabetización informacional para la Educación Superior (Framework for Information Literacy for Higher Education), amplía el alcance del concepto tradicional de la alfabetización informacional (determinar, acceder, ubicar, comprender, producir y usar información) hacia la producción colaborativa y el intercambio de información en entornos digitales participativos y en tecnologías emergentes, con énfasis en el pensamiento crítico y la metacognición (Mackey y Jacobson, 2011).

Finamente, se encuentra un solo modelo de carácter general que no es de alfabetización informacional, el cual se muestra en la tabla 4. El modelo ARCS es un método creado por Keller (2010) orientado hacia la motivación en entornos de aprendizaje y que comprende cuatro categorías conceptuales: atención, pertinencia, confianza y satisfacción. 
Tabla 4. Modelos que no son de alfabetización informacional

\begin{tabular}{|c|c|}
\hline $\begin{array}{c}\text { Modelos que no son de alfabetización informacio- } \\
\text { nal }\end{array}$ & Publicaciones \\
\hline Modelo ARCS (ARCS Model) & Chang y Chen (2015) \\
\hline
\end{tabular}

Fuente: elaboración propia

3.2 Objetivo específico 2: Determinar a través de qué modalidades de enseñanza y aprendizaje se ha aplicado la alfabetización informacional en la educación superior de tipo blended.

Considerando el tipo de estudio abordado, los documentos de la población estudiada se agrupan en siete categorías, que se muestran en la figura 1.

Figura 1. Tipos de estudios de las publicaciones estudiadas que representan las modalidades de enseñanza y aprendizaje en las que se ha aplicado la alfabetización informacional en la educación superior de tipo blended.

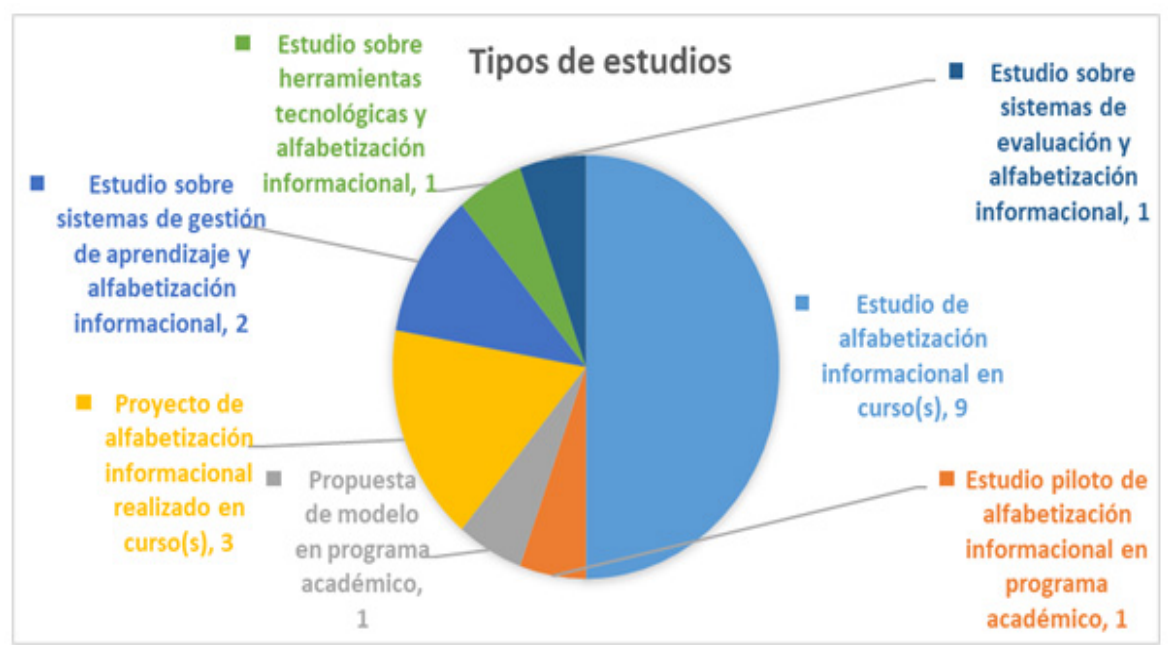

En la tabla 5 figura el detalle de los tipos de estudios, las modalidades, los principales conceptos estudiados en cada documento de investigación de la muestra y los autores de cada uno.

Tabla 5. Tipos de estudios, modalidades y principales conceptos estudiados

\begin{tabular}{|l|l|l|l|}
\hline Tipo de estudio & Modalidad & \multicolumn{1}{|c|}{ Principales conceptos estudiados } & \multicolumn{1}{c|}{ Documentos } \\
\hline & Curso & Habilidades de Al, modelo de Al & Chaiyama (2013) \\
\cline { 2 - 5 } Cursos & $\begin{array}{l}\text { Motivación de estudiantes, aprendizaje de Al, } \\
\text { modelo de aprendizaje }\end{array}$ & Chang y Chen (2015) \\
\cline { 2 - 5 } & Curso & $\begin{array}{l}\text { Diseño instruccional, habilidades de: } \\
\text { comunicación, Al y TIC }\end{array}$ & Zurita et al. (2015) \\
\cline { 2 - 5 } $\begin{array}{l}\text { Estudio de } \\
\text { alfabetización } \\
\text { informacional (AI) } \\
\text { en curso(s) }\end{array}$ & Cursos & $\begin{array}{l}\text { Sistemas de evaluación de Al, experiencia de } \\
\text { aprendizaje, pruebas de tipo ensayo, pruebas } \\
\text { automatizadas }\end{array}$ & $\begin{array}{l}\text { Nakayama, Yamamoto y } \\
\text { Santiago (2009) }\end{array}$ \\
\cline { 2 - 5 } & Curso & $\begin{array}{l}\text { Habilidades de comportamiento de la } \\
\text { información, uso de la información }\end{array}$ & Ata (2016) \\
\cline { 2 - 5 } & $\begin{array}{l}\text { Bibliotecas, cursos de Al en planes de } \\
\text { estudio, uso de herramientas de aprendizaje } \\
\text { electrónico, competencias en gestión de la } \\
\text { información }\end{array}$ & $\begin{array}{l}\text { Tonakiewicz-Kołosowska } \\
\text { et al. (2016) }\end{array}$ \\
\cline { 2 - 5 } & Curso & $\begin{array}{l}\text { Aprendizaje de Al, recomendaciones } \\
\text { personalizadas, retroalimentación aprendizaje } \\
\text { flexible }\end{array}$ & Peter et al. (2017) \\
\cline { 2 - 5 } & Curso & $\begin{array}{l}\text { Habilidades en Al, aprendizaje invertido } \\
\text { (flipped learning) }\end{array}$ & $\begin{array}{l}\text { Kurbanoĝlu y Akkoyunl } \\
\text { (2017) }\end{array}$ \\
\cline { 2 - 5 } & Curso & $\begin{array}{l}\text { Modelo de metaalfabetización (metaliteracy), } \\
\text { experiencia de aprendizaje }\end{array}$ & Ma et al. (2019) \\
\hline
\end{tabular}




\begin{tabular}{|c|c|c|c|}
\hline \multirow{3}{*}{$\begin{array}{l}\text { Proyecto de Al } \\
\text { realizado en } \\
\text { curso(s) }\end{array}$} & Curso & Habilidades de Al & Sult y Mills (2006) \\
\hline & Curso & Habilidades de Al, entorno wiki & $\begin{array}{l}\text { Niedbala y Fogleman } \\
\text { (2010) }\end{array}$ \\
\hline & Curso & Habilidades de Al, tablets & Kleinveldt y Zulu (2016) \\
\hline $\begin{array}{l}\text { Estudio piloto de } \\
\text { Al en programa } \\
\text { académico }\end{array}$ & $\begin{array}{l}\text { Programa } \\
\text { académico }\end{array}$ & $\begin{array}{l}\text { Habilidades de Al, modelo, entorno tecno- } \\
\text { lógico de aprendizaje, ciclo académico del } \\
\text { estudiante }\end{array}$ & Heinze y Schnurr (2008) \\
\hline $\begin{array}{l}\text { Propuesta } \\
\text { de modelo } \\
\text { en programa } \\
\text { académico }\end{array}$ & $\begin{array}{l}\text { Sistema } \\
\text { curricular }\end{array}$ & $\begin{array}{l}\text { Modelos de aprendizaje blended, ciclo } \\
\text { académico del estudiante }\end{array}$ & Mei et al. (2016) \\
\hline \multirow{2}{*}{$\begin{array}{l}\text { Estudio sobre } \\
\text { sistemas de } \\
\text { gestión de } \\
\text { aprendizaje y Al }\end{array}$} & $\begin{array}{l}\text { Diversos } \\
\text { LMS }\end{array}$ & Bibliotecas, LMS, enseñanza de Al & Lyon y Warlick (2013) \\
\hline & Liguides & $\begin{array}{l}\text { Contenidos alojados, Liguides, LMS, } \\
\text { destrezas y competencias de Al, } \\
\text { metaalfabetización }\end{array}$ & Mortimore y Baker (2019) \\
\hline $\begin{array}{l}\text { Estudio sobre } \\
\text { herramientas } \\
\text { tecnológicas y Al }\end{array}$ & $\begin{array}{l}\text { Herramientas } \\
\text { digitales en } \\
\text { internet }\end{array}$ & $\begin{array}{l}\text { Herramientas de tecnología educativa, } \\
\text { recursos educativos, instrucción de Al, } \\
\text { aprendizaje activo }\end{array}$ & Hess (2017) \\
\hline $\begin{array}{l}\text { Estudio sobre } \\
\text { sistemas de } \\
\text { evaluación y Al }\end{array}$ & $\begin{array}{l}\text { Sistema de } \\
\text { gestión de } \\
\text { evaluación } \\
\text { abierta en } \\
\text { línea }\end{array}$ & $\begin{array}{l}\text { Sistema de gestión de evaluación abierta en } \\
\text { línea, medición estandarizada, evaluación de } \\
\text { Al, evaluación de alfabetización de medios y } \\
\text { TIC }\end{array}$ & $\begin{array}{l}\text { Khlaisang y Koraneekij } \\
(2019)\end{array}$ \\
\hline
\end{tabular}

Fuente: elaboración propia

La primera categoría contiene nueve documentos que desarrollan algún tipo de estudio acerca de la alfabetización informacional en uno o más cursos. En todos los casos, se trata de cursos blended, a excepción del artículo de Tonakiewicz-Kołosowska et al. (2016) que analizan, además, cursos presenciales y en línea. Los conceptos más estudiados son los siguientes: habilidades o competencias de alfabetización informacional (5), aprendizaje de alfabetización informacional (2), experiencia de aprendizaje (2) y modelos de alfabetización informacional.

Los conceptos de habilidades (skills) o competencias son empleados en cinco documentos: Chaiyama (2013) pretende desarrollar un modelo para el aprendizaje blended que desarrolla habilidades de alfabetización informacional en estudiantes de educación física; Zurita et al. (2015) realizan un diseño instruccional en un curso blended sobre habilidades de alfabetización informacional, comunicación y alfabetización en TIC; Ata (2016) establece un diseño instruccional a ser utilizado en un entorno blended con el objetivo de mejorar las habilidades de comportamiento de la información en estudiantes de un curso de alfabetización informacional, mediante la interacción en un mundo virtual; Kurbanoĝlu y Akkoyunlu (2017) analizan los resultados de un curso en la modalidad de aprendizaje invertido (flipped learning) con el objetivo de brindar a los estudiantes destrezas en alfabetización informacional y medir el efecto de esta modalidad en su aprendizaje; finalmente, Tonakiewicz-Kołosowska et al. (2016) indagan cuál es la participación de las bibliotecas polacas universitarias en la implementación de cursos de alfabetización informacional en los planes de estudio y en qué medida se utiliza el aprendizaje electrónico; asimismo identifican las preferencias de las bibliotecas cuando utilizan herramientas de aprendizaje electrónico para desarrollar las competencias en gestión de la información.

En cuanto al concepto de aprendizaje en alfabetización informacional, Chang y Chen (2015) analizan la motivación de estudiantes en el aprendizaje de alfabetización informacional en un entorno blended mediante la aplicación del modelo ARCS, en tanto que Peter et al. (2017) pretenden mejorar el aprendizaje de alfabetización informacional mediante recomendaciones personalizadas en un entorno blended que comprende ocho capítulos con diversos recursos en línea y una sola sesión presencial luego de haber completado dichos capítulos, con el objetivo de que los estudiantes puedan manejar de forma flexible su propio aprendizaje de una manera eficiente.

La experiencia de aprendizaje es estudiada por Nakayama et al. (2009) y Ma et al. (2019). Los primeros analizan las pruebas de tipo ensayo como herramientas de evaluación para dos cursos blended y uno en línea, mediante un sistema de evaluación automatizada y la evaluación de expertos, y además, examinan la relación entre la experiencia del aprendizaje y los resultados del rendimiento en estudiantes japoneses. Los segundos incorporar el modelo de metalfabetización (metaliteracy) en un curso blended para analizar la experiencia de aprendizaje, los beneficios aprendizaje blended en el desarrollo de dicho modelo y los retos de la integración de las prácticas de metaalfabetización con el aprendizaje blended. 
Finalmente, los estudios de Chaiyama (2013) y Ma et al. (2019) desarrollan y aplican dos modelos de alfabetización informacional ya descritos en la sección anterior: modelo de gestión de aprendizaje blended para desarrollar habilidades de alfabetización informacional y metalfabetización (metaliteracy)

El segundo tipo de estudio corresponde a algún tipo de experiencia o proyecto de alfabetización informacional realizado en uno o más cursos. En los tres documentos el concepto principal es, al igual que en el punto anterior, el de habilidades de alfabetización informacional. Mientras que Sult y Mills (2006) proponen mejorar la instrucción y el desarrollo de habilidades de alfabetización informacional en un proyecto realizado en los años 2005 y 2006 en cursos de inglés, para Niedbala y Fogleman (2010) su objetivo es desarrollar habilidades de alfabetización informacional mediante un entorno wiki y un diseño instruccional elaborado con este fin. Asimismo, en esta línea, Kleinveldt y Zulu (2016) explican el proyecto que llevaron a cabo para asegurar que los estudiantes adquieran habilidades de alfabetización informacional a través del uso de tablets.

La tercera categoría está referida a un estudio piloto de alfabetización informacional en un programa académico — carrera-, llevado a cabo por Heinze y Schnurr (2008) con el objetivo de apoyar el desarrollo de habilidades de alfabetización informacional mediante un modelo —-modelo de andamiaje heurístico dentro del ciclo de vida del estudiante- y un entorno tecnológico de aprendizaje a lo largo del ciclo académico del estudiante.

La cuarta categoría se trata de una propuesta de modelos en un programa académico de tecnologías de la información en China a nivel del sistema curricular de ese país, que incluye el concepto de aprendizaje a lo largo del ciclo académico del estudiante, aplicado también por Heinze y Schnurr (2008). Los modelos de Mei et al. (2016) fueron descritos en la sección anterior.

Las siguientes categorías difieren de las anteriores ya que no están referidas a ningún curso o programa académico. La quinta categoría corresponde a estudios sobre sistemas de gestión de aprendizaje y alfabetización informacional. Así, Lyons y Warlick (2013) tratan de determinar cómo los bibliotecarios de ciencias de la salud incorporan los sistemas de gestión de contenidos para la enseñanza de la alfabetización informacional; en tanto que Mortimore y Baker (2019) desarrollan una propuesta para dos cursos blended sobre contenidos alojados en Liguides como sistema de gestión de aprendizaje, con el fin de promover destrezas y competencias de alfabetización informacional, según los conceptos centrales del Framework for Information Literacy for Higher Education (2015).

La sexta categoría es un estudio sobre herramientas tecnológicas y alfabetización informacional que propone el diseño de un conjunto de herramientas de tecnología educativa para la instrucción de alfabetización informacional mediante el aprendizaje activo (Hess, 2017).

Finalmente, la séptima categoría corresponde un estudio sobre sistemas de evaluación y alfabetización informacional que pretende desarrollar un sistema de gestión de evaluación abierta en línea, así como realizar una medición estandarizada para una evaluación de la alfabetización informacional, de medios y de TIC para estudiantes de educación superior (Khlaisang y Koraneekij, 2019).

\subsection{Objetivo específico 3: identificar qué entornos tecnológicos se han utilizado en la alfabetización informacional en la modalidad blended en educación superior}

A continuación, se presentan los entornos tecnológicos utilizados en las investigaciones realizadas, en dos de las cuales no se pudo identificar ningún entorno tecnológico. Los resultados se muestran en la figura 2.

Figura 2. Entornos tecnológicos que se han utilizado en la alfabetización informacional en la modalidad blended en educación superior según las publicaciones estudiadas.

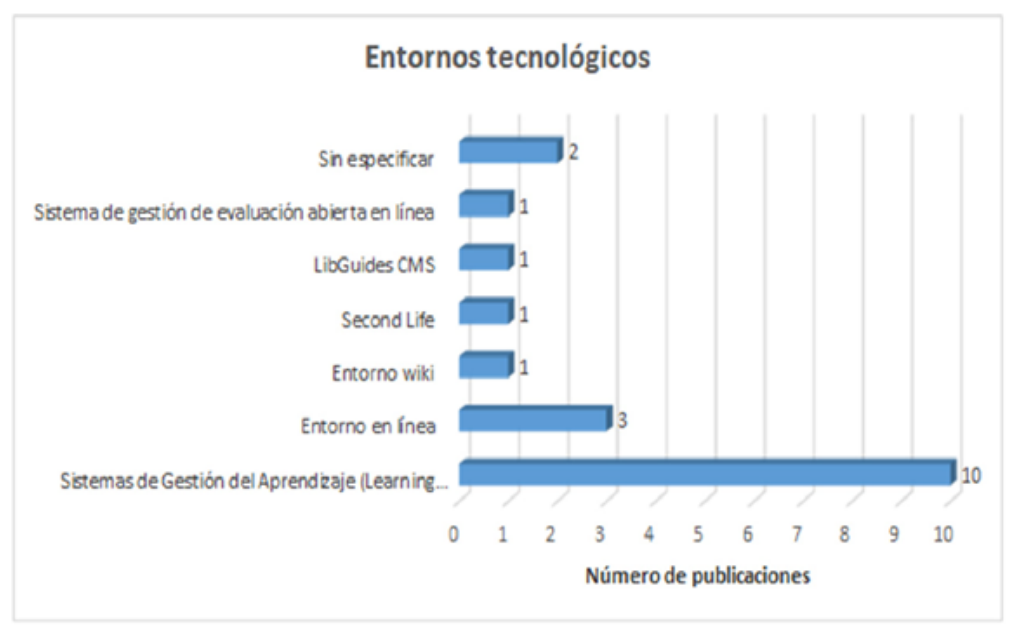


En primer lugar, están los sistemas de gestión del aprendizaje (10), que son entornos virtuales de aprendizaje que brindan un aula virtual en un ambiente web de forma segura, con la posibilidad de gestionar, incorporar contenidos y evaluar (Lyons y Warlick (2013). Entre los sistemas más mencionados destacan Blackboard (Ata, 2016; Kleinveldt y Zulu, 2016; Lyons y Warlick, 2013) y Desire2Learn D2L (Lyons y Warlick, 2013; Mortimore y Baker, 2019; Sult y Mills, 2006), seguidos por Moodle (Lyons y Warlick, 2013; Peter et al., 2017). Otros sistemas de gestión del aprendizaje son eCollege, Plateau y Sakai (Lyons y Warlick (2013). Además, los siguientes autores mencionan otros sistemas no especificados (Chaiyama, 2013; Chang y Chen, 2015; Tonakiewicz-Kołosowska et al., 2016; Zurita et al., 2015).

Los entornos virtuales de aprendizaje en línea proporcionan ambientes educativos que permiten a los estudiantes participar en actividades abiertas para mejorar sus habilidades cognitivas (Nykvist y Geva, 2016; Zanjani, Edwards,) y se relacionan con las tecnologías para el aprendizaje mediado por redes sociales y plataformas virtuales (Bustos y Coll (2010). En este sentido, pueden o no incorporar sistemas de gestión del aprendizaje.

Tres estudios se refieren a estos entornos. Nakayama et al. (2009) utilizan un entorno en línea que incluye videoclips, diapositivas utilizadas en clase y pruebas de evaluación en formato de opción múltiple. Por su parte, Hess (2017) propone un conjunto de aplicaciones web de tecnología educativa para la instrucción de alfabetización informacional, según la taxonomía de Bloom y con la justificación de su uso mediante los conceptos centrales del Framework for Information Literacy for Higher Education (2015). Asimismo, Kurbanoĝlu y Akkoyunlu (2017), con el fin de desarrollar las habilidades en alfabetización informacional en un curso en la modalidad de aprendizaje invertido (flipped learning), aplican un entorno virtual de aprendizaje que incluye diferentes recursos, como videotutoriales en Camtasia, PowToon, WordPress y página de Facebook para interacción entre estudiantes y docentes, entre otros.

Por otro lado, Niedbala y Fogleman (2010) utilizan un entorno wiki como espacio de trabajo compartido para los estudiantes y como repositorio de instrucciones, videotutoriales y otros materiales de apoyo, con el objetivo de desarrollar habilidades de alfabetización informacional.

En cuanto a la aplicación de mundos virtuales en la educación, Ata (2016) describe las experiencias de aprendizaje en una clase de alfabetización informacional mediante el uso de Second Life.

El uso de Libguides CMS como entorno virtual de aprendizaje es descrito por Mortimore y Baker (2019) como una plataforma colaborativa de creación de contenidos en dos cursos blended desarrollados por dos bibliotecarios.

Finalmente, cabe señalar el estudio de Khlaisang y Koraneekij (2019) sobre un sistema de gestión de evaluación abierta en línea, compatible con la plataforma Open edX, Moodle u otro sistema independiente para educación en línea, con el fin de medir tres tipos de alfabetización, entre las cuales está la informacional

\subsection{Objetivo específico 4: Reconocer en qué disciplinas se ha aplicado la alfabetización informacional en la modalidad blended en educación superior}

La figura 3 muestra las diferentes disciplinas en las que se han realizado las diferentes investigaciones estudiadas.

Figura 3. Disciplinas en las que se ha aplicado la alfabetización informacional en la modalidad blended en educación superior según las publicaciones estudiadas. 
Cabe resaltar que las investigaciones se realizan en una gran variedad de disciplinas de prácticamente todas las áreas del conocimiento. En primer lugar, figuran las disciplinas de ciencias de la información (Ata, 2016; Hess, 2017; Khlaisang y Koraneekij, 2019), y de inglés (Ma et al., 2019; Mortimore y Baker, 2019; Sult y Mills, 2006). A continuación, se encuentran las disciplinas de educación (Kurbanoĝlu y Akkoyunlu, 2017; Niedbala y Fogleman, 2010), y de tecnología/sociedad de la información (Mei et al., 2016; Nakayama et al., 2009). El resto de disciplinas se muestra en la tabla 6.

Tabla 1. Estándares de alfabetización informacional.

\begin{tabular}{|l|c|}
\hline \multicolumn{1}{|c|}{ Disciplinas } & Publicaciones \\
\hline Administración & Chang y Chen (2015) \\
\hline Ciencias de la computación & Ma et al. (2019) \\
\hline Ciencias de la salud (medicina y enfermería) & Chang y Chen (2015) \\
\hline Diseño & Chaiyama (2013) \\
\hline Educación física & Kleinveldt y Zulu (2016) \\
\hline General & Tonakiewicz-Kołosowska et al. (2016) \\
\hline Gestión ambiental & Chang y Chen (2015) \\
\hline Humanidades & Zurita et al. (2015) \\
\hline Ingeniería & Chang y Chen (2015) \\
\hline Ingeniería en información y control de gestión & Heinze y Schnurr (2008) \\
\hline Ingeniería eléctrica y de información & Peter et al. (2017) \\
\hline Medios de comunicación & \\
\hline Psicología & \\
\hline
\end{tabular}

Fuente: elaboración propia

\section{Discusión y conclusiones}

Los resultados muestran una gran variedad de hallazgos. Para empezar, a pesar de que la mayoría de documentos incorporan modelos o estándares de alfabetización informacional, solo dos desarrollan una propuesta de modelo adaptada al entorno blended (Chaiyama, 2013; Heinze y Schnurr, 2008) y uno introduce un modelo ya creado: la metaalfabetización (Ma et al., 2019). No obstante, los modelos forman parte, en mayor o menor medida, del contenido de las investigaciones. La Association of College and Research Libraries (ACRL) lidera la adopción de estándares, en especial los del año 2000, que son la base para los Estándares de Alfabetización Informacional Específicos de Psicología (2010) y constituyen la base de su evolución hacia el marco para la alfabetización en educación superior (ACRL, 2015).

Es de resaltar que este último constituye un modelo integrador y que tiene como uno de sus sustentos el ya mencionado modelo de metaalfabetización, presente también en Mortimore y Baker (2019) como criterio para la creación de contenidos en Libguides. En esta línea se encuentra el modelo KSAVE, que comprende un conjunto de conocimientos, habilidades y actitudes que son necesarias para desenvolverse en el siglo XXI. Aunque todavía son pocos los estudios sobre estos modelos más actuales e integradores, se espera que su adopción y la producción de investigaciones vayan en incremento, así como los estudios sobre modelos de alfabetización informacional a nivel de políticas de estado y su puesta en práctica, tal como Tonakiewicz-Kołosowska et al. (2016) y Chaiyama (2013).

En cuanto a las modalidades de enseñanza y aprendizaje, 14 documentos abordan estudios, proyectos realizados o estudios piloto de algún aspecto de la alfabetización informacional en cursos blended o en programas académicos. Varios de estos estudios abordan la planificación e implementación de un diseño curricular para mejorar o desarrollar habilidades de alfabetización informacional (Ata, 2016; Chaiyama, 2013; Heinze y Schnurr, 2008; Kleinveld y Zulu, 2016; Kurbanoĝlu y Akkoyunlu, 2017; Niedbala y Fogleman, 2010; Sult y Mills, 2006). Algunos de estos estudios involucran el desarrollo de otros procesos cognitivos como el pensamiento crítico (Ata, 2016; Heinze y Schnurr, 2008; Kleinveldt y Zulu, 2016; Mortimore y Baker, 2019; Sult y Mills, 2006), que resulta fundamental en los procesos de aprendizaje, desarrollo cognitivo y la búsqueda efectiva de información para la posterior toma de decisiones (Pinto, 2019). 
Otros conceptos importantes en el diseño instruccional para el desarrollo de habilidades de alfabetización informacional son el aprendizaje para toda la vida (Heinze y Schnurr, 2008; Tonakiewicz-Kołosowska et al., 2016) la colaboración y/o interacción (Ata, 2016; Kleinveldt y Zulu, 2016; Mortimore y Baker; Niedbala y Fogleman, 2010) y el aprendizaje significativo (Zurita et al., 2015).

De igual importancia para el aprendizaje autónomo en entornos blended, el concepto de retroalimentación es abordado directamente por Peter et al. (2017) mediante el diseño de un programa de ocho sesiones en línea a los que se les incorporó recomendaciones personalizadas en los contenidos en línea que los estudiantes debían completar según una prueba preliminar, con resultados positivos. En esta línea, Kurbanoĝlu y Akkoyunlu (2017) destacan, entre los resultados de su estudio de caso, que la retroalimentación es una de las partes más importantes de un aprendizaje efectivo que ayuda a los estudiantes a entender sus materias y otorgarles una guía de cómo mejorar su aprendizaje, por lo cual, en su investigación, la falta de retroalimentación en tiempo real constituyó uno de sus principales inconvenientes.

De igual forma, es deseable que se realicen investigaciones sobre otros aspectos emocionales o cognitivos que influyen en la alfabetización informacional en entornos blended, tal como la motivación según el modelo ARCS (Chang y Chen, 2015), cuyo estudio demostró que la participación de los estudiantes fue alentadora y satisfactoria, lo cual está en concordancia con Chen y Jang (2010), quienes, basados en la teoría de la autodeterminación y la motivación en el aprendizaje en línea, afirman que, para que los estudiantes afronten los problemas de aprendizaje, necesitan el apoyo constante de los instructores, compañeros y el personal administrativo.

En este sentido, los estudios futuros deberían contemplar estos conceptos que influyen directamente en el aprendizaje blended.

En cuanto a la participación de los bibliotecarios en el diseño o implementación de cursos o programas de alfabetización informacional, algunos estudios evidencian la importancia de su rol, tales como Kleinveldt y Zulu (2016), Lyons y Warlick (2013); Mortimore y Baker (2019), Sult y Mills (2006), y Tonakiewicz-Kołosowska et al. (2016).

En relación con los entornos tecnológicos empleados, la mayoría de ellos corresponden a sistemas de gestión del aprendizaje o entornos virtuales de aprendizaje en línea. Para la adecuada aplicación de un diseño instruccional en dichos entornos, algunos de los documentos estudiados mencionan los recursos y contenidos en cada sesión, tales como videoclips y diapositivas (Nakayama et al., 2009); textos descriptivos con capturas de pantalla, videos, conferencias, cuestionarios y documentos (Peter et al., 2017); aplicación Wordle.net de nubes de palabras, entrada de blog, formulario de evaluación, screencasts y aplicación QR para localizar libros de la biblioteca (Kleinveldt y Zulu, 2016); videotutoriales en Camtasia, aplicación PowToon, WordPress para compartir los videos y la plataforma Biloku para hacer disponibles los videos y las diapositivas (Kurbanoĝlu y Akkoyunlu, 2017). Sin embargo, el estudio más completo y de importancia para la enseñanza de la alfabetización informacional es el de Hess (2017) porque detalla diversos recursos en línea que se ajustan a los siguientes principios de Bloom aplicados a la información: recordar, entender, aplicar, analizar, evaluar y crear información, con el valor agregado de que justifica su inclusión mediante su alineamiento con los estándares de alfabetización informacional de educación superior de la ACRL (2015).

El uso de entornos virtuales de aprendizaje y de recursos virtuales en el aprendizaje blended implica necesariamente el aprendizaje de competencias digitales y de medios, la presencia del docente en la configuración del entorno en línea (Nortvig et al., 2018), así como un adecuado diseño curricular que tome en cuenta el contexto disciplinar (Pinto, 2019) y que considere los medios usados, que pueden o no estar limitados por la tecnología, tales como las herramientas de apoyo, el aprendizaje presencial, la colaboración virtual síncrona o asíncrona y el aprendizaje según el propio ritmo del estudiante (Kaur, 2013).

A partir de estos hallazgos se pueden considerar algunas líneas de investigación sobre los entornos tecnológicos.

Una primera línea de investigación es la aplicación de plataformas que no son propiamente sistemas de gestión de contenidos o entornos virtuales de aprendizaje y que han sido adaptados para el desarrollo de habilidades de alfabetización informacional, tales como Libguides (Mortimore y Baker, 2019), entorno wiki (Niedbala y Fogleman, 2010) y mundos virtuales (Ata, 2016).

Otra línea de investigación es el uso de redes sociales como componentes del entorno blended y la aplicación de los dispositivos móviles, tecnologías que apenas se han abordado en los documentos estudiados. 
En cuanto a las disciplinas estudiadas, a pesar del escaso número de documentos analizados, estas son variadas, por lo cual se espera tener estudios en otras disciplinas que no han sido abordadas, tales como artes, arquitectura o derecho.

Finalmente, se recomienda ampliar el alcance geográfico de las investigaciones y tener, por ejemplo, estudios en el ámbito de los países de América Latina, para lo cual, se debería considerar otras bases de datos como Google Académico.

\section{Referencias}

Adams, S., Cummins, M., Davis, A., Freeman, A., Hall Giesinger, C., y Ananthanarayanan, V. (2017). NMC Horizon Report: 2017 Higher Education Edition. Recuperado de https://library.educause.edu/-/media/files/ library/2017/2/2017horizonreporthe.pdf

Anderson, K., y May, F. (2010). Does the method of instruction matter? An experimental examination of information literacy instruction in the online, blended, and face-to-face classrooms. The Journal of Academic Librarianship, 36(6), 495-500. doi:10.1016/j.acalib.2010.08.005

Área, M., y Guarro, A. (2012). La alfabetización informacional y digital: fundamentos pedagógicos para la enseñanza y el aprendizaje competente. Revista Española De Documentación Científica, 35(Monográfico), 46-74. doi: 10.3989/redc.2012.mono.977

Arnold-Garza, S. (2014). The Flipped Classroom Teaching Model and its use for information literacy instruction. Communications in Information Literacy, 8(1), 7-22. doi:10.15760/comminfolit.2014.8.1.161

Association of College and Research Libraries (2015). Framework for Information Literacy for Higher Education. Recuperado de http://www.ala.org/acrl/standards/ilframework

Association of College and Research Libraries (2010). Psychology Information Literacy Standards. Recuperado de http://www.ala.org/acrl/standards/psych info lit

Association of College and Research Libraries (2000). Information Literacy Competency Standards for Higher Education. Recuperado de http://hdl.handle.net/11213/7668

Australian and New Zealand Institute for Information Literacy (2004). Australian and New Zealand Information Literacy Framework: principles, standards and practice. Recuperado de https://www.utas.edu.au/ data/assets/ pdf file/0003/79068/anz-info-lit-policy.pdf

Bartolomé, A., García-Ruiz, R., y Aguaded, I. (2018). Blended learning: panorama y perspectivas. Revista Iberoamericana de Educación a Distancia, 21(1), 33-56. doi: 10.5944/ried.21.1.18842

Brand-Gruwel, S., Wopereis, I., y Walraven, A. (2008). A descriptive model of information problem solving while using internet. --, 53(4). 1207-1217. doi:10.1016/j.compedu.2009.06.004

Big6 (2017) Big6 Skills Overview. Recuperado de https://thebig6.org/

Bustos, A., y Coll, C. (2010) Los entornos virtuales como espacio de enseñanza y aprendizaje: una perspectiva psicoeducativa para su caracterización y análisis. Revista Mexicana de Investigación Educativa, 163-184. Recuperado de http://ref.scielo.org/svdg58

Calderón, A. (2010). Informe APEI sobre alfabetización informacional. Recuperado de http://eprints.rclis. org/14972/1/Informeapeialfin.pdf

Castells, M. (2014). El impacto de internet en la sociedad: una perspectiva global. Recuperado de https://www. bbvaopenmind.com/wp-content/uploads/2014/03/BBVA-Comunicaci\%C3\%B3n-Cultura-Manuel-Castells-Elimpacto-de-internet-en-la-sociedad-una-perspectiva-global.pdf

Cedefop. (2017). Poland: European inventory on NQF 2016. Recuperado de https://www.cedefop.europa.eu/files/ poland - european inventory on naf 2016.pdf 
Chen, K. y Jang, S. (2010). Motivation in online learning: testing a model of self-determination theory. Computer in Human Behavior, 26, 741-752. doi:10.1016/j.chb.2010.01.011

Chen, L., Ritzhaupt, A. y Antonenko, P. (2019). Effects of the flipped classroom instructional strategy on students' learning outcomes: a meta-analysis. Educational Technology Research and Development, 67, 793-824. doi:10.1007/s11423-018-9633-7

Coonan, E., Geekie, J., Goldstein, S., Jeskins, L., Jones, R., Macrae-Gibson, R., ... Walton, Geof. (2018). CILIP Definition of Information Literacy 2018. Recuperado de https://infolit.org.uk/ILdefinitionCILIP2018.pdf

Crawford, J., y Irving, C. (2007). Information literacy: the link between secondary and tertiary education project and its wider implications. Journal of Librarianship and Information Science, 39(1), 17-26. doi:10.1177/0961000607074812

Cuevas, A. (2007). Lectura, alfabetización en información y biblioteca escolar. Gijón, España: Trea.

Day, R. (2005). Cómo escribir y publicar trabajos científicos. Washington D.C: Organización Panamericana de la Salud.

De Pablos, J. (2010). Universidad y sociedad del conocimiento. Las competencias informacionales y digitales. Revista de Universidad y Sociedad del Conocimiento, (7)2

Delgado López-Cózar, E., Robinson-García, N. y Torres-Salinas, D. (2014). The Google Scholar Experiment: how to index false papers and manipulate bibliometric indicators. Journal of the American Society for Information Science and Technology, 65(3): 446-454. doi:10.1002/asi.23056

Doğan, G., Şencan, I. y Tonta, Y. (2016). Does dirty data affect google scholar citations?. Proceedings of the American Society for Information Science and Technology, 53: 1-4. doi:10.1002/pra2.2016.14505301098

Eisenberg, M. y Berkowitz, R. (1990). Information Problem-solving: the Big Six Skills Approach to Library \& Information Skills Instruction. Norwood, New Jersey: Ablex Publishing Corporation

García Aretio, L. (2018). Blended learning y la convergencia entre la educación presencial y a distancia. Revista Iberoamericana de Educación a Distancia, 21(1), 9-22. doi: 10.5944/ried.21.1.19683

Giannasi-Kaimen, M. J., Carelli, A .E., y Gimenes da Cruz, V. A. (2010). Biblioteca digital y desarrollo de la competencia informacional: recursos y habilidades indispensables en la educación a distancia. En M, J. GiannasiKaimen, y A. E. Carelli. (Coords.), Recursos informacionales para compartir: acceso, disponibilidad y uso (pp. 167-191). Buenos Aires, Argentina: Alfagrama.

González, I. (2012). Necesidad de la alfabetización informacional en la educación superior. Revista de Comunicación Vivat Academia, 15(121), 65-76. Recuperado de https://dialnet.unirioja.es/descarga/articulo/5098314.pdf

Griffin, P. y Care, E. (2015). The ATC21S Method. En P. Griffin y E. Care (Eds.), Assessment and teaching of 21st Century skills. methods and approach (pp. 3-33). New York: Springer.

Gros, B., Garcia, I., y Escofet, A. (2012). Beyond the net generation debate: A comparison between digital learners in face-to-face and virtual universities. The International Review of Research in Open and Distributed Learning, 13(4), 190-210. doi:10.19173/irrodl.v13i4.1305

Jacso, P. (2005). As we may search-comparison of major features of the Web of Science, Scopus, and Google Scholar citation-based and citation-enhanced databases. Current Science, 89(9), 1537-1547. Recuperado de https://www.jstor.org/stable/24110924?seq=1

Jaramillo, P., Hennig, C. y Rincón, A. (2011). ¿Cómo manejan información los estudiantes de educación superior? El caso de la Universidad de La Sabana, Colombia. Información, Cultura y Sociedad, (25), 117-143. Recuperado de http://www.scielo.org.ar/pdf/ics/n25/n25a07.pdf

Kaur, M. (2013). Blended learning - its challenges and future. Procedia - Social and Behavioral Sciences, 93, 612-617. doi:10.1016/j.sbspro.2013.09.248

Keller, J. M. (2010). Motivational design for learning and performance: the ARCS model approach. New York: 
Springer.

Kurbanoĝlu, S. y Akkoyunlu, B. (2016). Information literacy and flipped learning. En D. Sales y M. Pinto (Eds.), Pathways into information literacy and communities of practice: teaching approaches and case studies (pp. 53-84). Cambridge, United States: Chandos Publishing.

Lanning, S. (2017). Concise guide to information literacy. Recuperado de https://slowrotation.memoryoftheworld. org/Scott $\% 20$ Lanning/Concise $\% 20$ Guide $\% 20$ to $\% 20$ Information $\% 20$ Liter $\% 20(31676$ )/Concise $\% 20$ Guide $\% 20$ to\%20Information $\% 20$ Li $\% 20-\% 20$ Scott $\% 20$ Lanning.pdf

Lau, J. y Cortés, J. (2009). Habilidades informativas: convergencia entre ciencias de información y comunicación. Comunicar, 16(32), 21-30. doi:10.3916/c32-2009-02-001

Lau, J. (2004). Directrices internacionales para la alfabetización informativa: propuesta. Recuperado de http:// online.ucn.cl/files/bidoc/Directrices DHI Propuesta.pdf

López, Meneses, E., Vázquez, Cano, E., y Román, P. (2015). Análisis e implicaciones del impacto del movimiento MOOC en la comunidad científica: JCR y Scopus (2010-13). Comunicar, 22(44), 73-80. doi:10.3916/C44-2015-08

Mackey, T. P. y Jacobson, T. E. (2011). Reframing information literacy as a metaliteracy. College and Research Libraries, (72)1, 62-78. Recuperado de https://crl.acrl.org/index.php/crl/article/view/16132/17578

Martín-Martín, A., Orduna-Malea, E. y Delgado López-Cózar, E. (2018). Coverage of highly-cited documents in Google Scholar, Web of Science, and Scopus: a multidisciplinary comparison. Scientometrics, 116, 2175-2188. doi:10.1007/s11192-018-2820-9

Martín-Martín, A., Orduna-Malea, E., Thelwall, M. y Delgado López-Cózar, E. (2018). Google Scholar, Web of Science, and Scopus: a systematic comparison of citations in 252 subject categories. Journal of Informetrics, 12(4), 1160-1177. doi:10.1016/J.JOI.2018.09.002

Merino-Trujillo, A. (2011). Como escribir documentos científicos (Parte 3). Artículo de revisión. Salud en Tabasco, 17(1-2), 36-40. Recuperado de http://www.redalyc.org/articulo.oa?id=48721182006

Nortvig, A. M., Petersen, A. K., y Balle, S. H. (2018). A literature review of the factors influencing e-learning and blended learning in relation to learning outcome, student satisfaction and engagement. The Electronic Journal of e-Learning, 16(1), 46-55. Recuperado de http://www.ejel.org/volume16/issue1/p46\#.W80JRwJhfnc.google

Orduna-Malea, E., Martín-Martín, A. y Delgado López-Cózar, E. (2017). Google Scholar as a source for scholarly evaluation: a bibliographic review of database errors. Revista Española de Documentación Científica, 40(4), e185. doi:10.3989/redc.2017.4.1500

Pinto, M. (2019). Evaluación de la alfabetización informacional en la educación superior: modelos, métodos e instrumentos. Buenos Aires, Argentina: Alfagrama.

Real Academia Española (2020). Modelo. Recuperado de https://dle.rae.es/modelo

REBIUN. Comisión mixta CRUE-TIC y REBIUN (2012). Competencias informáticas e informacionales (CI2) en los estudios de grado. Recuperado de http://hdl.handle.net/20.500.11967/62

Rodríguez, J. E. (2016). A massively flipped class-designing and implementing active learning information literacy instruction for a large enrollment course. Reference Services Review, 44(1), 4-20. doi:10.1108/RSR-07-2015-0033

Rovira, C., Codina, L., Guerrero-Solé, F. y Lopezosa, C. (2019). Ranking by Relevance and Citation Counts, a Comparative Study: Google Scholar, Microsoft Academic, WoS and Scopus. Future Internet, 11, 202. doi:10.3390/ fi11090202

Salinas, J., De Benito, B., Pérez, A., y Gisbert, M. (2018). Blended learning, más allá de la clase presencial. Revista Iberoamericana de Educación a Distancia, 21(1), 195-213. doi:10.5944/ried.21.1.18859

SCONUL Working Group on Information Literacy (2011). The SCONUL Seven Pillars of Information Literacy: Core 
Model For Higher Education. Recuperado de https://www.sconul.ac.uk/sites/default/files/documents/coremodel.pdf

Shah, S., Mahmood, K. y Hameed, A. (2017). Review of Google scholar, Web of Science, and Scopus search results: the case of inclusive education research. Library Philosophy and Practice, 1544. Recuperado de https:// digitalcommons.unl.edu/cgi/viewcontent.cgi?article=4419\&context=libphilprac

Singer, F. y Stoicescu, D. (2011). Using blended learning as a tool to strengthen teaching competences. Procedia Computer Science, 3, 1527-1531. doi:10.1016/j.procs.2011.01.043

Tuamsuk, K. (2013). Information literacy instruction in Thai higher education. Procedia - Social and Behavioral Sciences, 73, 145-150. doi:10.1016/j.sbspro.2013.02.034

United States. Institute of Education Sciences. (2019). ERIC Thesaurus: Standards. Recuperado de https://eric. ed.gov/?ti=Standards

Uribe, A. (2013). Lecciones aprendidas en programas de Alfabetización Informacional en universidades de Iberoamérica. Propuesta de buenas prácticas (tesis doctoral). Recuperado de http://hdl.handle.net/10760/22416

Wopereis I., Frerejean J. y Brand-Gruwel S. (2015). Information problem solving instruction in higher education: a case study on instructional design. En S. Kurbanoglu, J. Boustany, S. Špiranec, E. Grassian, D. Mizrachi y L. Roy (Eds.), Information Literacy: Moving Toward Sustainability. Third European Conference, ECIL 2015. Communications in Computer and Information Science (pp. 293-302). Switzerland: Springer International Publishing. doi:10.1007/978-3-319-28197-1_30

Zanjani, N., Edwards, S., Nykvist, S. y Geva, S. (2016). LMS Acceptance: the Instructor role, The Asia-Pacific Education Researcher, 25(4) 519-526. doi:10.1007/s40299-016-0277-2

\section{Anexo 1}

Ata, R. (2016). An exploration of higher education teaching in Second Life in the context of blended learning. Turkish Online Journal of Educational Technology, 15(3), 9-26.

Chaiyama, N. (2015). The Development of Blended Learning Management Model in Developing Information Literacy Skills (BL-ILS Model). International Journal of Information and Education Technology, 5(7), 483-489. doi:10.7763/IJIET.2015.V5.554

Chang, N.-C., Chen y H.-H. (2015). A motivational analysis of the ARCS model for information literacy courses in a blended learning environment. Libri, 65(2), 129-142. doi: 10.1515/libri-2015-0010

Heinze, N. y Schnurr, J.-M. (2008). Developing information literacy skills by using e-learning environments in higher education. En D. Remenyi (Ed.), Proceedings of the 7th European Conference on e-Learning, ECEL, (pp. 492498). Reading, United Kingdom: Academic Publishing Limited.

Hess, A.N. (2016). Using theory and practice to build an instructional technology tool kit. En T. Maddison y M. Kumaran (Eds.), Distributed learning: pedagogy and technology in online information literacy instruction (pp. 4766). Cambridge, United States: Chandos Publishing.

Khlaisang, J. y Koraneekij, P. (2019). Open online assessment management system platform and instrument to enhance the information, media, and ICT literacy skills of 21st century learners. International Journal of Emerging Technologies in Learning, 14(7), 111-127. doi:10.3991/ijet.v14i07.9953

Kleinveldt, L.T. y Zulu, M. (2016). Integrating tablet technology into information literacy training at CPUT libraries: a pilot project. Library Hi Tech News, 33(4), 10-14. doi:10.1108/LHTN-12-2015-0083

Kurbanoĝlu, S. y Akkoyunlu, B. (2016). Information literacy and flipped learning. En D. Sales y M. Pinto (Eds.), Pathways into information literacy and communities of practice: teaching approaches and case studies (pp. 53-84). Cambridge, United States: Chandos Publishing.

Lyons, T. y Warlick, S. (2013). Health sciences information literacy in CMS environments: learning from our peers. Electronic Library, 31(6), 770-780. doi:10.1108/EL-06-2012-0063 
Ma, J., Li, C. y Liang, H.-N. (2019). Enhancing students' blended learning experience through embedding metaliteracy. Education Research International, 1-8. doi:10.1155/2019/6791058

Mei, L., Qi, L. y Zhang, Y. (2016). Blended learning models for information technology education as general course for college students. En F. Lee Wang, T. Lam Wong, O. Au, Q. Liu y D. Wu (Eds.), Proceedings - 2015 International Symposium on Educational Technology, (pp. 47-51.). Los Alamitos, California, United States: IEEE Computer Society. doi: 10.1109/ISET.2015.18

Mortimore, J.M. y Baker, R.L. (2018). Supporting student-led content creation in the distance learning environment with Libguides CMS. Journal of Library and Information Services in Distance Learning. doi:10.1080/153329 $0 X .2018 .1499239$

Nakayama, M., Yamamoto, H., y Santiago, R. (2009). The role and use of essay tests in e-learning: a Japanese case study. Electronic Journal of e-Learning, 8(2), 173-178.

Niedbala, M.A. y Fogleman, J. (2010). Taking library 2.0 to the next level: Using a course wiki for teaching information literacy to honors students. Journal of Library Administration, 50(7), 867-882. doi:10.1080/01930826.2010.488986

Peter, J., Leichner, N., Mayer, A.-K., y Krampen, G. (2017). Making information literacy instruction more efficient by providing individual feedback. Studies in Higher Education, 42(6). doi:10.1080/03075079.2015.1079607

Sult, L. y Mills, V. (2006). A blended method for integrating information literacy instruction into English composition classes. Reference Services Review, 34(3), 368-388. doi:10.1108/00907320610685328

Tonakiewicz-Kołosowska, A., Socik, I. y Gajewska, M. (2016). Information competencies and their implementation in the educational process of Polish universities exploratory studies. En R. M. Idrus y N Zainuddin (Eds.), Proceedings of the 11th International Conference on e-Learning (pp. 223-226). Reading, United Kingdom: Academic Conferences and Publishing International.

Zurita, G., Hasbun, B., Baloian, N. y Jerez, O. (2015). A blended learning environment for enhancing meaningful learning using 21 st century skills. En G. Chen, V. Kumar, Kingshuk, R. Huang y S.C. Kong (Eds.), Emerging issues in smart learning, (pp.1-8). Berlin, Germany: Springer. 


\section{Dados dos autores}

Andre Armel Maguiña Ballón

Licenciado en Letras con amplios conocimientos en Ciencias de la Información e Historia. Su experiencia y formación en Bibliotecología y Ciencias de la Información le permite llevar a cabo la organización y automatización de una unidad de información, así como la diseminación de la información y el tratamiento de la misma por medios digitales. Actualmente, se desempeña como especialista en orientación editorial, publicaciones académicas y servicios de apoyo a la producción intelectual, enseñanza y aprendizaje.

andre.maguina@gmail.com

Received: 2020-04-10

Accepted: $2020-10-25$

\section{$(c)$ EY}

This work is licensed under a Creative Commons Attribution 4.0

United States License.

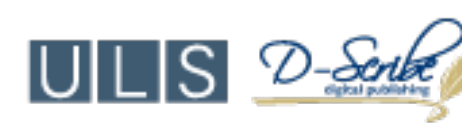

This journal is published by the University Library System of the University of Pittsburgh as part of its D-Scribe Digital Publishing Program and is cosponsored by the University of Pittsburgh Press 